\title{
Fixador externo tipo delta no manejo pós-operatório de retalhos microcirúrgicos no membro inferior: Experiência de um hospital terciário*
}

\section{External Delta Fixator in the Postoperative Management of Microsurgical Flaps in the Lower Limb: Experience of a Tertiary Hospital}

\author{
Bruno Moraes Vasconcelos ${ }^{1}$ \\ Amanda Favaro Cagnolati2 ${ }^{20}$ \\ 1 Programa de Cirurgia da Mão do Departamento de Ortopedia e \\ Traumatologia do Hospital das Clínicas da Faculdade de Medicina de \\ Ribeirão Preto da Universidade de São Paulo (HC-FMRP-USP), \\ Ribeirão Preto, SP, Brasil \\ 2 Divisão de Cirurgia da Mão do Departamento de Ortopedia, \\ Traumatologia e Anestesiologia do Hospital das Clínicas da \\ Faculdade de Medicina de Ribeirão Preto da Universidade de São \\ Paulo (HC-FMRP-USP), Ribeirão Preto, SP, Brasil \\ 3 Professor Titular e Chefe da Divisão de Cirurgia da Mão do \\ Departamento de Ortopedia, Traumatologia e Anestesiologia do \\ Hospital das Clínicas da Faculdade de Medicina de Ribeirão Preto da \\ Universidade de São Paulo (HC-FMRP-USP), Ribeirão Preto, SP, Brasil \\ Rev Bras Ortop 2022;57(1):69-74.
}

Mario Matiotti Neto ${ }^{10}$ Luis Guilherme Rosifini Alves Rezende ${ }^{20}$ Alex Eduardo Calderón Irusta2 ${ }^{2}$ Nilton Mazzer ${ }^{3}$ (i)

Endereço para correspondência Luis Guilherme Rosifini Alves Rezende, MD, Hospital das Clínicas, Faculdade de Medicina de Ribeirão Preto, Universidade de São Paulo. Av. Bandeirantes 3.900Vila Monte Alegre, Ribeirão Preto, SP, 14049-900, Brasil (e-mail: Igrezende@usp.br).

\begin{abstract}
Resumo
Palavras-chave

- retalho miocutâneo

- retalho perfurante

- microcirurgia

- fraturas ósseas

Objetivo Avaliar o uso de fixadores externos, na configuração kickstand do tipo delta, como método adjuvante no período pós-operatório de pacientes submetidos a retalhos livres nos membros inferiores.

Métodos Ao todo, 17 fixadores externos do tipo delta foram utilizados em pacientes submetidos a retalhos livres nos membros inferiores. A técnica cirúrgica foi realizada de forma padronizada, com o pino distal localizado $6 \mathrm{~cm}$ proximal à anastomose, e o pino proximal, $6 \mathrm{~cm}$ distal à tuberosidade anterior da tíbia.

Resultados A idade média da amostra foi de 34,76 anos (variação: 15 a 66 anos). Foram selecionados 11 homens e 6 mulheres. Utilizou-se a artéria tibial posterior em 14 casos, e a tibial anterior, em 3 casos. O tempo médio de uso dos fixadores externos foi de 3,88 semanas. A taxa de reoperação foi de $17,64 \%$; a de perda do retalho foi de $11,76 \%$; a de sucesso foi de $88,23 \%$; e a taxa de infecção foi de $5,9 \%$.
\end{abstract}

Trabalho realizado no Hospital das Clínicas, Faculdade de Medicina de Ribeirão Preto, Universidade de São Paulo, Ribeirão Preto, SP, Brasil. recebido

29 de Dezembro de 2020

aceito

07 de Abril de 2021

Publicado on-line

Outubro 25, 2021
DOI https://doi.org/ $10.1055 / \mathrm{s}-0041-1735832$. ISSN 0102-3616. (c) 2021. Sociedade Brasileira de Ortopedia e Traumatologia. All rights reserved.

This is an open access article published by Thieme under the terms of the Creative Commons Attribution-NonDerivative-NonCommercial-License, permitting copying and reproduction so long as the original work is given appropriate credit. Contents may not be used for commercial purposes, or adapted, remixed, transformed or built upon. (https://creativecommons.org/ licenses/by-nc-nd/4.0/)

Thieme Revinter Publicações Ltda., Rua do Matoso 170, Rio de Janeiro, RJ, CEP 20270-135, Brazil 


\begin{abstract}
Keywords

- myocutaneous flap

- perforator flap

- microsurgery

- fractures, bone

Objective To evaluate the use of external fixators in the delta-type kickstand configuration as an adjuvant method in the postoperative period of patients submitted to free flaps in the lower limbs.

Methods A total of 17 external delta fixators were used in patients submitted to free flaps in the lower limbs. The surgical technique was performed in a standardized manner, with the distal pin located $6 \mathrm{~cm}$ proximally to the anastomosis, and the proximal pin, $6 \mathrm{~cm}$ distally to the anterior tuberosity of the tibia.

Results The mean age of the sample was of 34.76 years (range: 15 to 66 years). In total, 11 men and 6 women were selected. The posterior tibial artery was used in 14 cases, and the anterior tibial artery, in 3 cases. The mean time of use of the external fixators was of 3.88 weeks. The rate of reoperation was of $17.64 \%$; that of retail loss was of $11.76 \%$; that of success rate was of $88.23 \%$; and the rate of infection was of $5.9 \%$. Conclusion The use of delta-type fixators as an adjunct method in the postoperative period is reliable; however, more studies are needed to evaluate its true role in the postoperative period.
\end{abstract}

Conclusão $\mathrm{O}$ uso de fixadores do tipo delta como método adjuvante no pósoperatório é confiável; porém, mais estudos são necessários para avaliar seu verdadeiro papel no pós-operatório.

\section{Introdução}

Os avanços nas técnicas microcirúrgicas permitiram o manejo das falhas de cobertura nos membros inferiores, o que provocou uma diminuição das taxas de amputação e proporcionou melhor funcionalidade do membro afetado., ${ }^{1,2}$ A maioria das causas de falhas de cobertura nos membros inferiores são: traumáticas, oncológicas, infecciosas ou vasculares. $\mathrm{O}$ objetivo final da cirurgia reconstrutiva dos membros inferiores é a restauração funcional do membro, sendo necessárias estabilização óssea, cobertura adequada das partes moles, ausência de dor, e uma sensibilidade protetora do membro. ${ }^{2,3}$

Apesar da evolução técnica e do aperfeiçoamento da prática microcirúrgica, complicações ainda são relatadas. Estudos retrospectivos ${ }^{4,5}$ demonstram séries de casos com alta variabilidade nas taxas de sucesso e complicações. Uma metanálise de Xiong et al. ${ }^{5}$ (2016) demonstrou taxas de complicações de $6 \%$ de perda do retalho livre, $6 \%$ de trombose vascular e necrose parcial da pele, $4 \%$ de formação de hematoma, e $4 \%$ de deiscência da ferida operatória. Por esta razão, técnicas adjuvantes ao manejo do retalho microcirúrgico são essenciais para o sucesso da cirurgia reconstrutiva de salvamento do membro. ${ }^{6}$

A utilização dos métodos de fixação externa para elevar o membro e evitar os efeitos compressivos sobre o retalho é um conceito já descrito. Entretanto, diferentes configurações do fixador externo foram desenvolvidas para facilitar sua aplicabilidade, oferecer maior estabilidade ao membro, permitir procedimentos ósseos concomitantes, e aliviar compressões externas adicionais sobre o retalho. ${ }^{6,7}$ Outrossim, o uso de fixadores externos facilita 0 acesso ao retalho para a monitori- zação da viabilidade pós-operatória e de reabordagens cirúrgicas posteriores, conforme necessidade, além de facilitar trocas de curativos, mudanças de decúbito, trocas de vestimenta, e proporcionar posturas mais confortáveis aos pacientes. ${ }^{7,8}$

O objetivo deste estudo foi avaliar o uso de fixadores externos do tipo delta como método adjuvante no período pós-operatório de pacientes submetidos a retalhos livres para falhas de cobertura nos membros inferiores.

\section{Materiais e Métodos}

Estudo retrospectivo realizado pela revisão de prontuários de pacientes que foram submetidos ao uso do fixador externo tipo delta após a confecção do retalho microcirúrgico livre para falha de cobertura nos membros, entre março de 2018 e março de 2019. Este trabalho foi aprovado pelo comitê de ética sob o número CAAE 14510719.4.0000.5440.

As variáveis sexo, idade e comorbidades prévias foram avaliadas, assim como o local da lesão e sua etiologia. Lesões de origem traumática foram diferenciadas pelo grau da lesão de partes moles, ao passo que lesões de origem tumoral foram avaliadas pelo grau histológico do tumor. As variáveis referentes aos procedimentos foram o tipo de retalho microcirúrgico realizado e o vaso receptor para a anastomose. A necessidade de intervenção de salvamento no leito e seu procedimento, bem como a necessidade de reabordagem no centro cirúrgico, foram documentadas. 0 tempo total de fixação externa também foi avaliado.

Os critérios de inclusão foram pacientes com falha de cobertura no terço distal da perna em uso de fixador externo do tipo delta para a manutenção de retalhos microcirúrgicos livres. Foram excluídos pacientes que não concordaram em 


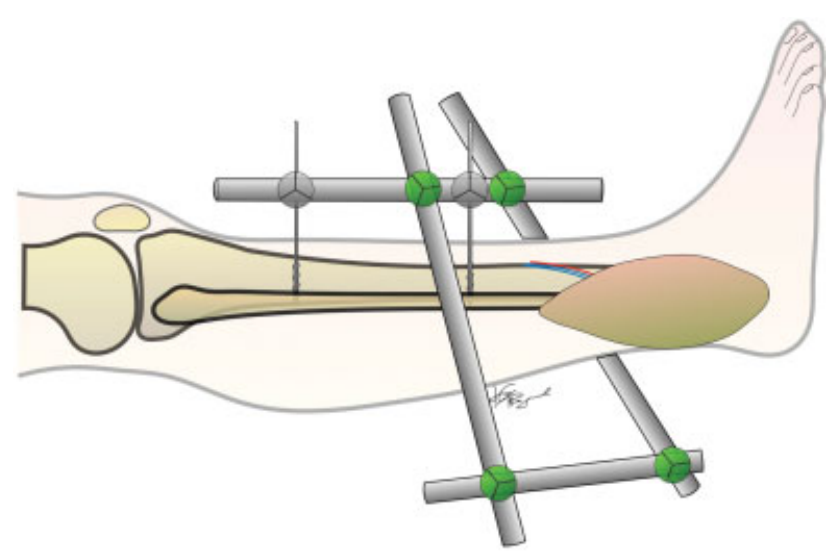

Fig. 1 Fixador externo do tipo delta, na configuração kickstand.

participar e pacientes submetidos a cobertura por retalhos pediculados.

\section{Técnica Cirúrgica}

O paciente foi submetido a raquianestesia com sedação e posicionado em decúbito dorsal em mesa cirúrgica radiotransparente. O procedimento de instalação do fixador externo iniciou-se após a conclusão do retalho microcirúrgico. O membro foi posicionado em extensão, e a fluoroscopia foi utilizada para auxiliar na instalação do fixador externo.

Instalou-se um fixador externo modular de tíbia que continha 2 hastes de carbono de comprimento grande (400 mm), 2 hastes de carbono de comprimento médio $(300 \mathrm{~mm}), 2$ pinos Schanz autorrosqueantes de 5,0 $\mathrm{mm}$ de diâmetro, 2 conectores tubo-pino, e 4 conectores tubo a tubo.

Optou-se pelo posicionamento dos pinos de Schanz perpendiculares à porção anteromedial da tíbia para a instalação do fixador externo, respeitando a zona de segurança da perna, e utilizando o local da anastomose vascular como ponto de referência. O primeiro pino de schanz (pino distal) foi instalado $6 \mathrm{~cm}$ proximal ao sítio da anastomose vascular microcirúrgica, e o segundo pino (pino proximal) foi colocado $6 \mathrm{~cm}$ distal à tuberosidade anterior da tíbia, conforme observado na - Figura 1. Um conector do tipo tubo-pino foi colocado em cada pino de Schanz, e os pinos foram interligados por uma haste de comprimento médio, orientada longitudinalmente na região anteromedial da perna, configurando um sistema linear uniplanar.

Dois conectores do tipo tubo a tubo foram acoplados no sistema linear, e duas hastes de comprimento grande foram direcionadas para a porção distal do membro. Uma haste foi posicionada no lado medial, e a outra, no lado lateral, mantendo-se uma distância entre as barras e a porção distal do membro de aproximadamente $6 \mathrm{~cm}$. Ao final, foi instalada a haste posterior, de tamanho médio, com dois conectores tubo a tubo nas extremidades, conectando as hastes medial e lateral, respeitando a altura de $6 \mathrm{~cm}$ de distância do membro, e dando aspecto final ao fixador externo tipo delta com uma distância circunferencial de $6 \mathrm{~cm}$ entre o retalho microcirúrgico e as barras (-Figura 2).

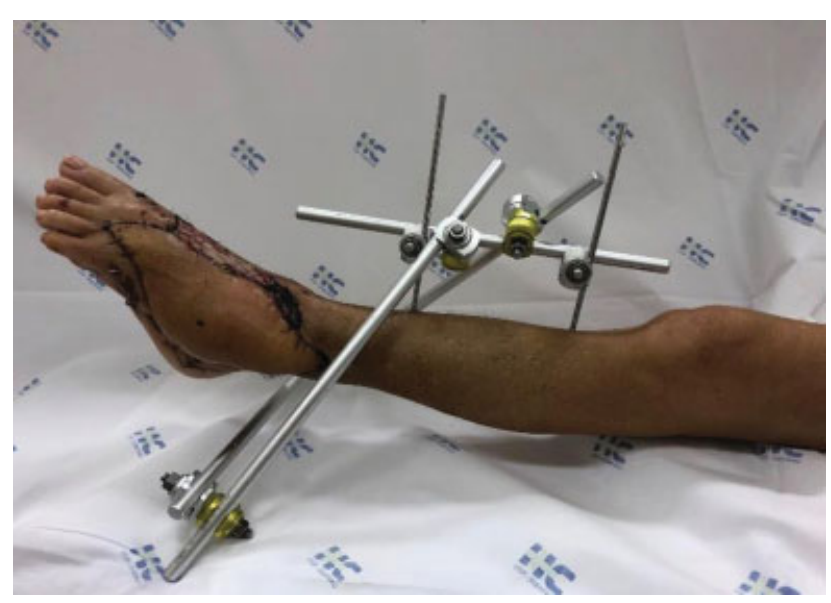

Fig. 2 Fixador externo do tipo delta instalado em paciente, com boa cicatrização da pele.

\section{Resultados}

A amostra foi composta por 15 pacientes submetidos a 17 procedimentos microcirúrgicos com utilização do fixador externo tipo delta. Em dois pacientes, foi necessária a realização de dois retalhos microcirúrgicos, devido a extensas falhas de cobertura. Os 17 procedimentos foram analisados separadamente.

Destes procedimentos, 11 foram realizados em pacientes do sexo masculino, e 6 , em pacientes do sexo feminino. A faixa etária média da amostra foi de 34,76 anos (variação: 15 a 66 anos). Ao todo, 5 pacientes apresentavam comorbidades. As comorbidades dos pacientes e os dados epidemiológicos encontram-se disponíveis na - Tabela 1.

Apenas um caso não tinha falha de cobertura de origem traumática. Nos casos traumáticos, 10 foram por fraturas expostas do tipo IIIA, e 6, por fraturas expostas do tipo IIIB. 0 padrão das fraturas e a localização topográfica das falhas de cobertura encontram-se disponíveis na - Tabela 2.

Em relação aos retalhos livres utilizados, foram treze anterolaterais da coxa, dois grandes dorsais, e dois do tipo chinês (do antebraço baseado na artéria radial). Os vasos receptores foram a artéria tibial posterior em 14 casos, e a tibial anterior em 3 casos.

Três casos necessitaram de intervenção no leito para afrouxamento de curativo. Já em três outros casos, houve a necessidade de reabordagem de emergência no centro cirúrgico, sendo um para drenagem de hematoma, e os outros dois para abordagem do pedículo. As causas de reabordagem do pedículo foram trombose arterial, secundária a infecção em um caso. O paciente com trombose arterial secundária a infecção foi manejado com desbridamento do retalho, antibióticos endovenosos, submetido a um novo retalho microcirúrgico livre, e obteve bom resultado. No outro caso de trombose arterial, optou-se pelo novo retalho do tipo grande dorsal, sendo obtida a cobertura adequada. Ambos permaneceram com o uso do fixador externo como método adjuvante de manejo pós-operatório. Estes dados estão disponíveis na - Tabela 3.

O tempo médio de permanência do fixador externo foi de 3,88 semanas (variação: 1 a 7 semanas). Alguns destes 
72 Fixador externo no manejo pós-operatório de retalhos microcirúrgicos no membro inferior Vasconcelos et al.

Tabela 1 Características dos pacientes e aspectos epidemiológicos

\begin{tabular}{|c|c|c|c|c|c|c|c|}
\hline Caso & Idade (anos) & Sexo & Comorbidades & Trauma & Local da falha & Fratura & $\begin{array}{l}\text { Classificação da } \\
\text { fratura exposta }\end{array}$ \\
\hline 1 & 25 & $\mathrm{~F}$ & Não & Não & Posterior & Tumoral & - \\
\hline 2 & 60 & $\mathrm{~F}$ & HAS, DP & Sim & Medial & Lisfranc & IIIB \\
\hline 3 & 60 & $\mathrm{~F}$ & HAS, DP & Sim & Lateral & Lisfranc & IIIA \\
\hline 4 & 20 & $\mathrm{M}$ & Não & Sim & Lateral & Tálus-cuboide & IIIA \\
\hline 5 & 48 & $\mathrm{M}$ & Não & Sim & Calcâneo & Calcâneo & IIIA \\
\hline 6 & 37 & $\mathrm{M}$ & Não & Sim & $\mathrm{PL}$ & Tornozelo & IIIA \\
\hline 7 & 38 & $\mathrm{M}$ & Não & Sim & $\mathrm{PL}$ & Tornozelo & IIIA \\
\hline 8 & 24 & $\mathrm{M}$ & Não & Sim & Lateral & Pilão-calcâneo & IIIA \\
\hline 9 & 15 & $\mathrm{M}$ & Não & Sim & PL + calcâneo & Pilão-calcâneo & IIIB \\
\hline 10 & 42 & $\mathrm{~F}$ & HAS, TBG & Sim & $\mathrm{PL}$ & Tornozelo & IIIB \\
\hline 11 & 66 & $\mathrm{~F}$ & HAS, DL, HP & Sim & Lateral & Tornozelo & IIIB \\
\hline 12 & 32 & $\mathrm{M}$ & TBG & Sim & Posterior & Tornozelo & IIIB \\
\hline 13 & 23 & $\mathrm{M}$ & Não & Sim & Posterior & Tornozelo & IIIB \\
\hline 14 & 41 & $\mathrm{M}$ & Não & Sim & Medial + calcâneo & Calcâneo & IIIA \\
\hline 15 & 21 & $\mathrm{~F}$ & Não & Sim & Medial & Pilão-calcâneo & IIIA \\
\hline 16 & 19 & $\mathrm{M}$ & Não & Sim & Lateral & Pilão & IIIA \\
\hline 17 & 20 & $\mathrm{M}$ & Não & Sim & $P L$ & Pilão & IIIA \\
\hline
\end{tabular}

Abreviaturas: DL, dislipidemia; DP, depressão; F, feminino; HAS, hipertensão arterial sistêmica; HP, hipotireoidismo; M, masculino; PL, posterolateral .

pacientes necessitaram de enxertia de pele tardia ou aguardaram granulação final de pequenas porções de pele adjacente ao retalho. A taxa de reoperação foi de $17,64 \%$; a de perda do retalho, de 11,76\%; a de infecção, de 5,9\%; e a taxa de sucesso do retalho foi de $88,23 \%$.

\section{Discussão}

A imobilização pós-operatória é indispensável em cirurgias reconstrutivas para falhas de cobertura nos membros inferiores; entretanto, deve-se permitir a visualização e o monitoramento contínuo do retalho. O uso de talas oferece vantagens quanto à praticidade e ao custo; todavia, as inúmeras desvantagens limitam o seu uso rotineiro. ${ }^{9}$

Buford e Trzeciak ${ }^{9}$ descreveram o uso de fixadores externos de Hoffman para a imobilização do tornozelo e manuseio de retalhos livres em três pacientes, sem relatar complicações. Ênfase foi dada à facilidade de monitorização do retalho, bem como à eliminação do efeito compressivo, de odor, e à ausência de colonização bacteriana. Em contraste a este estudo, os autores demonstram a fixação estendida aos metatarsianos para a manutenção da articulação do tornozelo em dorsiflexão e a prevenção do equino no retropé. Neste estudo, não foram observadas complicações como rigidez do tornozelo ou equino, pois os pacientes realizaram fisioterapia motora para a amplitude de movimento do tornozelo e do pé durante o período no qual utilizaram o fixador externo. Fixadores externos do tipo delta na configuração kickstand foram instalados após a osteossíntese e o manejo das fraturas e lesões ósseas, não tendo sido utilizados para o manejo ósseo.
A técnica descrita neste estudo é de simples execução e com boa reprodutibilidade. Além dos benefícios citados, o uso do fixador externo apresenta importante vantagem como método adjuvante no tratamento de fraturas

Tabela 2 Retalhos e vasos receptores

\begin{tabular}{|l|l|l|}
\hline Caso & Tipo de retalho & Vaso receptor \\
\hline 1 & ALT & TP \\
\hline 2 & ALT & TP \\
\hline 3 & ALT & TA \\
\hline 4 & ALT & TP \\
\hline 5 & ALT & TP \\
\hline 6 & GD & TP \\
\hline 7 & ALT & TP \\
\hline 8 & ALT & TP \\
\hline 9 & ALT & TP \\
\hline 10 & Chinês & TP \\
\hline 11 & ALT & TP \\
\hline 12 & ALT & TP \\
\hline 13 & GD & TP \\
\hline 14 & ALT & TA \\
\hline 15 & ALT & TP \\
\hline 16 & ALT & TP \\
\hline 17 & Chinês & TA \\
\hline
\end{tabular}

Abreviaturas: ALT, anterolateral da coxa; GD, grande dorsal; TA, artéria tibial anterior; TP, artéria tibial posterior. 
Tabela 3 Resultados e complicações

\begin{tabular}{|l|l|l|l|l|l|l|l|l|}
\hline Caso & $\begin{array}{l}\text { Intervenção } \\
\text { no leito }\end{array}$ & $\begin{array}{l}\text { Tipo de } \\
\text { intervenção }\end{array}$ & Reabordagem & $\begin{array}{l}\text { Causa da } \\
\text { reabordagem }\end{array}$ & $\begin{array}{l}\text { Perda do } \\
\text { retalho }\end{array}$ & $\begin{array}{l}\text { Motivo da } \\
\text { perda }\end{array}$ & $\begin{array}{l}\text { Tempo } \\
\text { de FE } \\
\text { (semanas) }\end{array}$ & $\begin{array}{l}\text { Procedimento } \\
\text { adicional }\end{array}$ \\
\hline 1 & Não & Não & Não & Não & Não & Não & 4 & Não \\
\hline 2 & Não & Não & Não & Não & Não & Não & 4 & Não \\
\hline 3 & Não & Não & Não & Não & Não & Não & 5 & Não \\
\hline 4 & Não & Não & Sim & Sangramento & Não & Não & 7 & Granulação \\
\hline 5 & Não & Não & Sim & Infeç̧ão & Sim & Infeç̧ão & 1 & Novo retalho \\
\hline 6 & Não & Não & Sim & $\begin{array}{l}\text { Insuficiência } \\
\text { arterial }\end{array}$ & Sim & $\begin{array}{l}\text { Trombose } \\
\text { arterial }\end{array}$ & 1 & Novo retalho \\
\hline 7 & Sim & $\begin{array}{l}\text { Afrouxar } \\
\text { Curativo }\end{array}$ & Não & Não & Não & Não & 4 & $\begin{array}{l}\text { llizarov } \\
\text { (transporte) }\end{array}$ \\
\hline 8 & Não & Não & Não & Não & Não & Não & 4 & Enxerto de pele \\
\hline 9 & Não & Não & Não & Não & Não & Não & 3 & Não \\
\hline 10 & Não & Não & Não & Não & Não & Não & 5 & Enxerto de pele \\
\hline 11 & Não & Não & Não & Não & Não & Não & 3 & Não \\
\hline 12 & Não & Não & Não & Não & Não & Não & 4 & Não \\
\hline 13 & Não & Não & Não & Não & Não & Não & 4 & Não \\
\hline 14 & Sim & $\begin{array}{l}\text { Afrouxar } \\
\text { Curativo }\end{array}$ & Não & Não & Não & Não & 3 & Não \\
\hline 15 & Sim & $\begin{array}{l}\text { Afrouxar } \\
\text { Curativo }\end{array}$ & Não & Não & Não & Não & 5 & Enxerto de pele \\
\hline 16 & Não & Não & Não & Não & Não & Não & 4 & Enxerto de pele \\
\hline 17 & Não & Não & Não & Não & Não & Não & 5 & Enxerto de pele \\
\hline
\end{tabular}

Abreviatura: FE, fixador externo.

complexas, muitas vezes presentes em concomitância com falhas de cobertura cutânea. Sua montagem é a mesma, independentemente do fabricante específico. O objetivo do fixador externo é evitar pontos compressivos, estase vascular, medidas de controle do edema, além de auxiliar nos cuidados pós-operatórios em pacientes politraumatizados. ${ }^{10}$ $\mathrm{O}$ aspecto final do retalho e do membro após a retirada do fixador externo pode ser observado na - Figura 3.

O suporte com fixador externo eliminou o fator compressivo e foi capaz de manter a elevação adequada do membro no pós-operatório, sendo dispensáveis as medidas de resgate no leito. $\mathrm{O}$ incremento de um sítio seguro para evitar forças

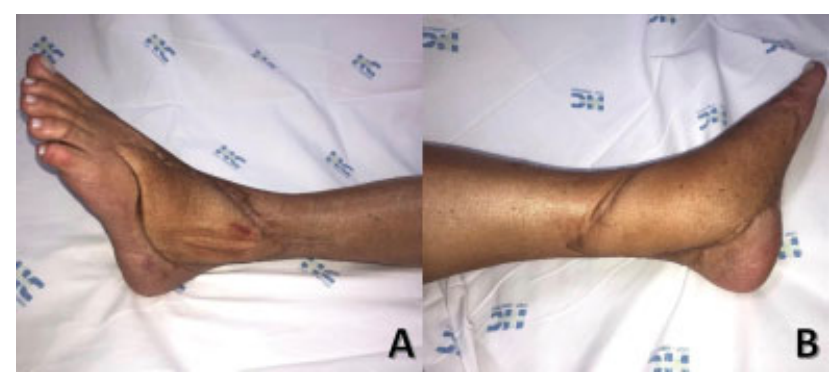

Fig. 3 Resultado pós-operatório após a retirada. (A) Caso $\mathrm{n}^{\circ} 2$, com retalho anterolateral da coxa para falha de cobertura na região lateral. (B) Caso $n^{\circ} 3$, com retalho anterolateral da coxa para falha de cobertura na região medial. de cisalhamento sobre o retalho é a chave no manejo pósoperatório. $^{11}$

Castro-Aragon, Rapley e Trevino ${ }^{10}$ utilizaram o fixador externo do tipo kickstand em 11 falhas de cobertura, e explicaram que em todos os casos não houve alterações na pele; porém, não relataram as possíveis complicações do método. Outros estudos ${ }^{11-13}$ descreveram o seu uso e as modificações nas montagens, mas com séries menores, como o estudo de Kachare et al., ${ }^{11}$ que descreve o sucesso na obtenção da cicatrização em uma série de quatro pacientes, sem relatar complicações.

Estudos anteriores ${ }^{6,10-13}$ retrataram os benefícios do emprego de fixadores externos no manejo de retalhos ou lesões na região posterior do retropé e sua oportuna aplicabilidade neste grupo de pacientes. Isto justifica-se pela ampla necessidade de cuidados pós-operatórios na monitoração dos retalhos. ${ }^{14-19}$

O posicionamento pós-operatório também tem se mostrado um fator importante no prognóstico dos retalhos, tendo em vista que a compressão do pedículo pode acarretar complicações, inclusive a perda do retalho. ${ }^{20-22}$ De forma geral, a taxa de não sobrevivência dos retalhos livres no cenário agudo do trauma pode chegar a $12 \%$. Taxas mais baixas de perda do retalho, como $4 \%$, também foram observadas, o que nos mostra a necessidade de cuidados pósoperatórios específicos. ${ }^{23-28}$ 
Maruccia et al. ${ }^{29}$ descreveram uma série de 14 pacientes, na qual relataram como complicação apenas a infecção em 2 pacientes, e com um tempo médio de 3,79 semanas (variação: 3 a 5 semanas) de uso do fixador externo. Os autores não replataram rigidez ou deformidade em equino nos tornozelos. A maioria dos estudos não registraram a taxa de viabilidade dos retalhos, apesar de relatarem um número de procedimentos menor ao apresentado neste estudo, e utilizarem o fixador externo do tipo kickstand para o manejo de feridas pelo uso de curativo a vácuo, retalhos pediculados, e também retalhos livres. ${ }^{9-13,29}$

As limitações deste estudo são o pequeno grupo de pacientes, as poucas variáveis disponíveis, bem como a falta de grupo de controle e de comparações com outros estudos.

\section{Conclusão}

Este estudo demonstrou uma série de casos na qual o emprego do fixador externo, como método adjuvante nos cuidados pós-operatórios dos retalhos microcirúrgicos para falhas de coberturas dos membros inferiores, demonstrou ser um método eficiente. Entretanto, não podemos afirmar que a sua utilização está correlacionada de forma pontual com o sucesso do procedimento microcirúrgico, visto que outras variáveis não foram mensuradas e ponderadas neste estudo. Mais estudos são necessários, com uma série maior de retalhos e com um grupo de controle para definir a aplicabilidade e a vantagem do uso de fixadores externos como método adjuvante de manejo pós-operatório.

\section{Suporte Financeiro}

Não houve suporte financeiro de fontes públicas, comerciais, ou sem fins lucrativos.

\section{Conflito de Interesses}

Os autores declaram não haver conflito de interesses.

\section{Referências}

1 Wei F, Al Deek NF, Lin Tay SK. Principals and techniques of microvascular surgery. In: Plastic Surgery: Principles. Philadelphia: Elsevier;2018:444-472

2 Ninkovic M, Voigt S, Dornseifer U, Lorenz S, Ninkovic M. Microsurgical advances in extremity salvage. Clin Plast Surg 2012;39 (04):491-505

3 Benacquista T, Kasabian AK, Karp NS. The fate of lower extremities with failed free flaps. Plast Reconstr Surg 1996;98(05):834-840

4 Perrot P, Bouffaut AL, Perret C, Connault J, Duteille F. Risk factors and therapeutic strategy after failure of free flap coverage for lower-limb defects. J Reconstr Microsurg 2011;27(03):157-162

5 Xiong L, Gazyakan E, Kremer T, et al. Free flaps for reconstruction of soft tissue defects in lower extremity: A meta-analysis on microsurgical outcome and safety. Microsurgery 2016;36(06): 511-524

6 Zgonis T, Roukis TS. Off-loading large posterior heel defects after sural artery soft-tissue flap coverage with stacked taylor spatial frame foot plate system. Oper Tech Orthop 2006;16(01):32-37

7 Nappi JF, Drabyn GA. External fixation for pedicle-flap immobilization: a new method providing limited motion. Plast Reconstr Surg 1983;72(02):243-245
8 Zgonis T, Stapleton JJ. Innovative techniques in preventing and salvaging neurovascular pedicle flaps in reconstructive foot and ankle surgery. Foot Ankle Spec 2008;1(02):97-104

9 Buford GA, Trzeciak MA. A novel method for lower-extremity immobilization after free-flap reconstruction of posterior heel defects. Plast Reconstr Surg 2003;111(02):821-824

10 Castro-Aragon OE, Rapley JH, Trevino SG. The use of a kickstand modification for the prevention of heel decubitus ulcers in trauma patients with lower extremity external fixation. J Orthop Trauma 2009;23(02):145-147

11 Kachare SD, Vivace BJ, Henderson JT, et al. Kickstand External Fixator for Immobilization Following Free Flap Plantar Calcaneal Reconstruction. Eplasty 2019;19:e11

12 Berkowitz MJ, Kim DH. Using an external fixation "kickstand" to prevent soft-tissue complications and facilitate wound management in traumatized extremities. Am J Orthop 2008;37(03):162-164

13 Roukis TS, Landsman AS, Weinberg SA, Leone E. Use of a hybrid "kickstand" external fixator for pressure relief after soft-tissue reconstruction of heel defects. J Foot Ankle Surg 2003;42(04):240-243

14 Hirigoyen MB, Urken ML, Weinberg H. Free flap monitoring: a review of current practice. Microsurgery 1995;16(11):723-726, discussion 727

15 Baumeister SP, Spierer R, Erdmann D, Sweis R, Levin LS, Germann GK. A realistic complication analysis of 70 sural artery flaps in a multimorbid patient group. Plast Reconstr Surg 2003;112(01): 129-140, discussion 141-142

16 Bhatnagar A. Miniwatermattress to prevent pressure sores over the heel. Plast Reconstr Surg 1997;99(03):927-928

17 Yilmaz M, Karatas O, Barutcu A. The distally based superficial sural artery island flap: clinical experiences and modifications. Plast Reconstr Surg 1998;102(07):2358-2367

18 Clark J, Mills JL, Armstrong DG. A method of external fixation to offload and protect the foot following reconstruction in high-risk patients: the SALSAstand. Eplasty 2009;9:e21

19 Singh B, Cordeiro PG, Santamaria E, Shaha AR, Pfister DG, Shah JP. Factors associated with complications in microvascular reconstruction of head and neck defects. Plast Reconstr Surg 1999;103 (02):403-411

20 Noack N, Hartmann B, Küntscher MV. Measures to prevent complications of distally based neurovascular sural flaps. Ann Plast Surg 2006;57(01):37-40

21 Price MF, Capizzi PJ, Watterson PA, Lettieri S. Reverse sural artery flap: caveats for success. Ann Plast Surg 2002;48(05):496-504

22 Hanasono MM, Skoracki RJ, Yu P. A prospective study of donor-site morbidity after anterolateral thigh fasciocutaneous and myocutaneous freeflap harvest in 220 patients. Plast Reconstr Surg 2010;125:e209-e214

23 Godina M. Early microsurgical reconstruction of complex trauma of the extremities. Plast Reconstr Surg 1986;78(03):285-292

24 Karanas YL, Nigriny J, Chang J. The timing of microsurgical reconstruction in lower extremity trauma. Microsurgery 2008; 28(08):632-634

25 Coskunfirat OK, Chen HC, Spanio S, Tang YB. The safety of microvascular free tissue transfer in the elderly population. Plast Reconstr Surg 2005;115(03):771-775

26 Griffin JR, Thornton JF. Lower extremity reconstruction. SRPS 2003;9(37):1-31

27 Byrd HS, Cierny G 3rd, Tebbetts JB. The management of open tibial fractures with associated soft-tissue loss: external pin fixation with early flap coverage. Plast Reconstr Surg 1981;68(01):73-82

28 Sun TB, Chien SH, Lee JT, Cheng LF, Hsu LP, Chen PR. Is dextran infusion as an antithrombotic agent necessary in microvascular reconstruction of the upper aerodigestive tract? J Reconstr Microsurg 2003;19(07):463-466

29 Maruccia M, Elia R, Caizzi G, et al. Free flap and kickstand external fixator in foot and ankle soft tissue reconstruction. The versatility of a microsurgical-friendly application of an orthopedic device. Injury 2018;49(Suppl 3):S105-S109 